\title{
Bounds on the response time under control constraints
}

\author{
S. T. Glad and A. J. Isaksson* \\ Department of Electrical Engineering \\ Linköping University, SE-581 83 Linköping, Sweden \\ WWW: http://www.control.isy.liu.se \\ Email: torkel@isy.liu.se \\ * Department of Signals, Sensors and Systems \\ Royal Institute of Technology, SE-100 44 Stockholm, Sweden \\ Email: alf.isaksson@s3.kth.se
}

May 4, 1999

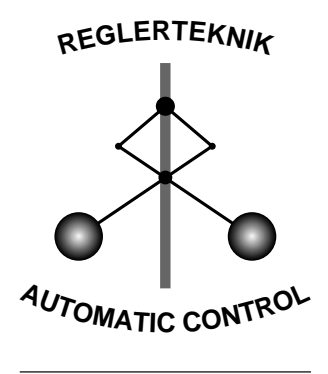

LINKÖPING

Report no.: LiTH-ISY-R-2141

Submitted to 37 th CDC

Technical reports from the Automatic Control group in Linköping are available by anonymous ftp at the address ftp.control.isy.liu.se. This report is contained in the compressed postscript file $2141 . \mathrm{ps} . \mathrm{Z}$. 



\title{
Bounds on the response time under control constraints
}

\author{
S. T. Glad* and A. Isaksson** \\ * Dept. of Electrical Engineering \\ Linköping University \\ SE-581 83 Linköping, Sweden \\ torkel@isy.liu.se \\ ** Dept. of Signals Sensors and Systems \\ Royal Institute of Technology \\ SE-100 44 Stockholm, Sweden \\ alf.isaksson@s3.kth.se
}

May 4, 1999

\begin{abstract}
We consider the problem of transferring the output of a linear system from one equilibrium value to another under control amplitude constraint. It is possible to give a simple lower bound on the time required, expressed in the longest time constant, the gain of the system, the change in $y$ and the control bound.
\end{abstract}

\section{Introduction}

Recently much interest has been paid to the various system properties that restrict performance. The references [1], [3], [4], [5] all discuss the basic performance limitations. A common practical control design problem is to find the best possible rise time or settling time for a step response when the control amplitude is limited. To use the criteria in the references mentioned above, one would scale the variables to reflect the amplitude constraints and then transform the time domain properties into bandwidth or gain crossover properties. We are looking for more direct time domain criteria. For that purpose we consider a single-input-single-output system with transfer function $G(s)$ and minimal realization

$$
\dot{x}=A x+B u, \quad y=C x
$$

We assume the initial condition $x(0)=0$, and that we want to transfer the state to a new equilibrium

$$
y\left(t_{f}\right)=C x\left(t_{f}\right)=y_{0}, \quad \dot{x}\left(t_{f}\right)=0
$$

without exceeding the control constraint

$$
|u(t)| \leq u_{0}
$$


The question is: what is the smallest $t_{f}$ ? The answer is of course obtained by solving the minimum time optimal control problem, see e. g. [2], but we are looking for some simple lower bound. We do that by first reviewing the minimum time solution for a first order system and then extending the result to higher order systems. We also consider the case when $G$ contains an integrator separately.

\section{Minimum time control of a first order system}

Consider the system

$$
\dot{x}=-\frac{1}{\tau} x+\frac{k}{\tau} u, \quad|u| \leq u_{0}
$$

Suppose one wants to transfer the state from $x(0)=0$ to $x\left(t_{f}\right)=y_{0}>0$ with the smallest possible value of $t_{f}$. This is a minimum time problem and it is easy to see from the maximum principle that the solution is to use the constant control $u=u_{0}$ until the desired state is reached. It is then necessary that $u_{0}>\bar{u}=\frac{y_{0}}{k}$. From the equation

$$
y_{0}=x\left(t_{f}\right)=\int_{0}^{t_{f}} e^{-\left(t_{f}-\sigma\right) / \tau} \frac{k u_{0}}{\tau} d \sigma
$$

it follows that

$$
t_{f}=-\tau \log \left(1-\frac{y_{0}}{k u_{0}}\right)=-\tau \log \left(1-\frac{\bar{u}}{u_{0}}\right)
$$

We see that the minimum time is proportional to the system time constant and depends critically on $\bar{u} / u_{0}$, i.e. the ratio between the control required to maintain the new state and the maximum control available. If the variables are scaled so that $u_{0}=y_{0}=1$, then the minimum time is given by

$$
t_{f}=-\tau \log \left(1-\frac{1}{k}\right)
$$

\section{A lower bound on the minimum time control of a general linear system}

Now consider a general linear SISO system with transfer function $G(s)$ or in state space form

$$
\begin{aligned}
& \dot{x}=A x+B u, \quad|u| \leq u_{0} \\
& y=C x
\end{aligned}
$$

where the dimension of the state vector $x$ is $n>1$. We pose the same problem: to transfer the state from $x(0)=0$ to an equilibrium $x\left(t_{f}\right)$, such that $y\left(t_{f}\right)=$ $C x\left(t_{f}\right)=y_{0}$, with the smallest possible $t_{f}$.

Suppose the system has a real negative pole $-1 / \tau$. Then it is possible to perform a coordinate change so that (8) can be written in the block triangular 
form

$$
\begin{aligned}
\frac{d}{d t}\left[\begin{array}{l}
x_{r} \\
x_{d}
\end{array}\right] & =\left[\begin{array}{cc}
A_{11} & A_{12} \\
0 & -\frac{1}{\tau}
\end{array}\right]\left[\begin{array}{l}
x_{r} \\
x_{d}
\end{array}\right]+\left[\begin{array}{c}
B_{1} \\
\frac{k}{\tau}
\end{array}\right] u \\
y & =\left[\begin{array}{ll}
C_{1} & c_{2}
\end{array}\right]\left[\begin{array}{l}
x_{r} \\
x_{d}
\end{array}\right]
\end{aligned}
$$

where $x_{d}, k$ and $c_{2}$ are scalars, $x_{r}$ is an $n-1$-vector, and $A_{11}, A_{12}$ and $B_{1}$ are matrices of appropriate dimensions. We assume that the mode $x_{d}$ is controllable, so that $k \neq 0$. The desired final state satisfies the following equations

$$
\begin{aligned}
0 & =A_{11} x_{r}\left(t_{f}\right)+A_{12} x_{d}\left(t_{f}\right)+B_{1} \bar{u} \\
0 & =-\frac{1}{\tau} x_{d}\left(t_{f}\right)+\frac{k}{\tau} \bar{u} \\
y_{0} & =C_{1} x_{r}\left(t_{f}\right)+c_{2} x_{d}\left(t_{f}\right)
\end{aligned}
$$

where $\bar{u}$ is the control needed to maintain the steady state. The task of transferring the state from $x(0)=0$ to $x\left(t_{f}\right)$ satisfying $y_{0}=C x\left(t_{f}\right)$ then requires the subtask of transferring $x_{d}$ from $x_{d}=0$ to $x_{d}(t f)$ satisfying (13), i.e. $x_{d}\left(t_{f}\right)=k \bar{u}$. This subtask is of the form described in section 2 and the minimum time according to $(6)$ is

$$
t_{f, x_{d}}=-\tau \log \left(1-\frac{x_{d}\left(t_{f}\right)}{k u_{0}}\right)=-\tau \log \left(1-\frac{\bar{u}}{u_{0}}\right)
$$

The time for the transfer of the complete state is then bounded below by this value:

$$
t_{f} \geq-\tau \log \left(1-\frac{\bar{u}}{u_{0}}\right)
$$

If the static gain is finite, then $\bar{u}=y_{0} / G(0)$, so it is also possible to write

$$
t_{f} \geq-\tau \log \left(1-\frac{y_{0}}{G(0) u_{0}}\right)
$$

If the variables are scaled so that $u_{0}=1, y_{0}=1$, then

$$
t_{f} \geq-\tau \log \left(1-\frac{1}{G(0)}\right)
$$

\section{The case of infinite static gain}

The formulas of the preceding section are not useful if $G$ has infinite static gain. Suppose $G$ has a single pole at the origin. We can then modify (10) and (11) to

$$
\begin{aligned}
\frac{d}{d t}\left[\begin{array}{l}
x_{r} \\
x_{d}
\end{array}\right] & =\left[\begin{array}{cc}
A_{11} & A_{12} \\
0 & 0
\end{array}\right]\left[\begin{array}{l}
x_{r} \\
x_{d}
\end{array}\right]+\left[\begin{array}{c}
B_{1} \\
k
\end{array}\right] u \\
y & =\left[\begin{array}{ll}
C_{1} & c_{2}
\end{array}\right]\left[\begin{array}{l}
x_{r} \\
x_{d}
\end{array}\right]
\end{aligned}
$$


We assume controllability of the pole at the origin so that $k \neq 0$. The final state then satisfies the following relations

$$
\begin{aligned}
0 & =A_{11} x_{r}\left(t_{f}\right)+A_{12} x_{d}\left(t_{f}\right)+B_{1} \bar{u} \\
0 & =k \bar{u} \\
y_{0} & =C_{1} x_{r}\left(t_{f}\right)+c_{2} x_{d}\left(t_{f}\right)
\end{aligned}
$$

Since there is no other pole at the origin, $A_{11}$ is nonsingular and

$$
y_{0}=\left(-C_{1} A_{11}^{-1} A_{12}+c_{2}\right) x_{d}\left(t_{f}\right)
$$

The minimum time to transfer the integrator

$$
\dot{x}_{d}=k u
$$

from the origin to the final state

$$
\frac{y_{0}}{-C_{1} A_{11}^{-1} A_{12}+c_{2}}
$$

is then

$$
t_{f, x_{d}}=\frac{y_{0}}{\left(-C_{1} A_{11}^{-1} A_{12}+c_{2}\right) k u}
$$

This is then a lower bound on the minimum time $t_{f}$. Defining

$$
g_{1}=\lim _{s \rightarrow 0} s G(s)
$$

and noting that

$$
g_{1}=\left(-C_{1} A_{11}^{-1} A_{12}+c_{2}\right) k
$$

we get the expression

$$
t_{f} \geq \frac{y_{0}}{g_{1} u_{0}}
$$

\section{Tightness of the lower bound}

We have given the lower bounds (17), (24) on the time of transfer from $y=0$ to $y=y_{0}$. To investigate how tight this bound is we investigate a system with two time constants

$$
\begin{aligned}
\frac{d}{d t}\left[\begin{array}{l}
x_{1} \\
x_{2}
\end{array}\right] & =\left[\begin{array}{cc}
-1 / \tau_{1} & 0 \\
0 & -1 / \tau_{2}
\end{array}\right]\left[\begin{array}{l}
x_{1} \\
x_{2}
\end{array}\right]+\left[\begin{array}{l}
b_{1} \\
b_{2}
\end{array}\right] u \\
y & =\left[\begin{array}{ll}
c_{1} & c_{2}
\end{array}\right]\left[\begin{array}{l}
x_{1} \\
x_{2}
\end{array}\right]
\end{aligned}
$$

The minimum time problem is solved by a control of the form

$$
\begin{aligned}
& u=u_{0}, \quad 0 \leq t \leq t_{1} \\
& u=-u_{0}, \quad t_{1}<t \leq t_{f}
\end{aligned}
$$


Substituting this control into the system equations gives the following condition on the final time $t_{f}$

$$
2^{1-\nu}\left(1+k^{-1}+e^{-t_{f} / \tau_{2}}\right)^{\nu}-e^{-t_{f} / \tau_{1}}-1-k^{-1}=0
$$

where $\nu=\tau_{2} / \tau_{1}$ and $k=G(0) u_{0} / y_{0}$. This equation can be solved numerically to give the true value of $t_{f}$, which can then be compared with the lower bound (17). The ratio of the true value of $t_{f}$ and the estimated lower bound is given in figure 1 as a function of $k$ for the case $\tau_{1}=1, \tau_{2}=0.1$. The corresponding

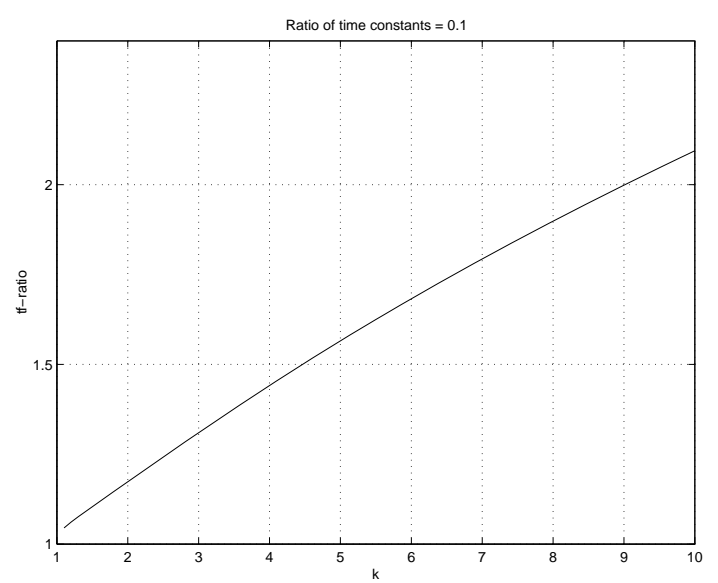

Figure 1: Ratio of true to estimated value of $t_{f}$ as a function of $k$ for $\tau_{1}=1$, $\tau_{2}=0.1$.

figure for $\tau_{1}=1, \tau_{2}=0.01$ is given in figure 2 . In figure 3 the minimum time

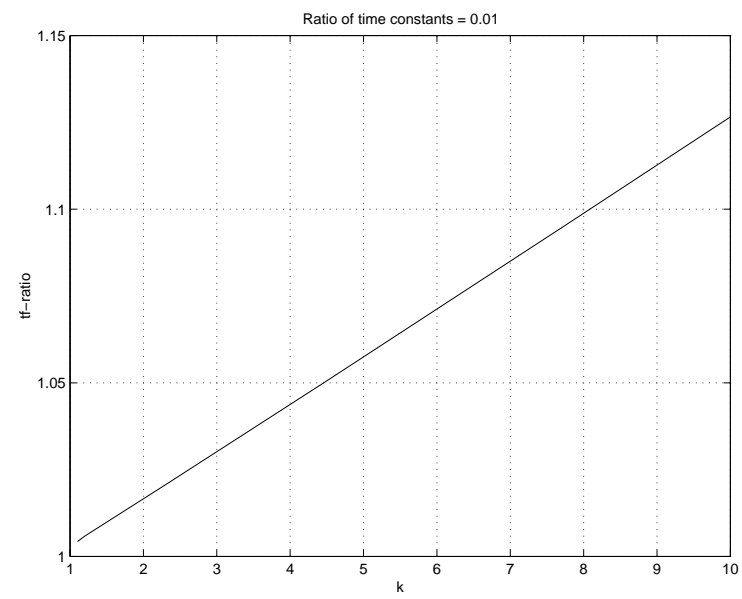

Figure 2: Ratio of true to estimated value of $t_{f}$ as a function of $k$ for $\tau_{1}=1$, $\tau_{2}=0.01$. 
response of the system

$$
G(s)=\frac{9}{(s+1)(0,1 s+1)}
$$

with the constraint $|u| \leq 1$ is given. The lower bound according to (17), is 0.12 in this case. If the system gain is lowered so that

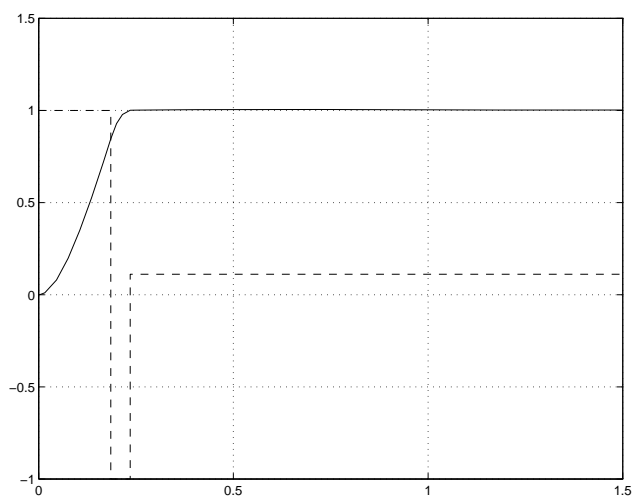

Figure 3: Minimum time step response. $G(0)=9, u_{0}=y_{0}=1$, time constants 1 and 0.1 .

$$
G(s)=\frac{1.5}{(s+1)(0,1 s+1)}
$$

the response is as shown in figure 4. Equation (17) gives the lower bound $1.1 \mathrm{in}$ this case.

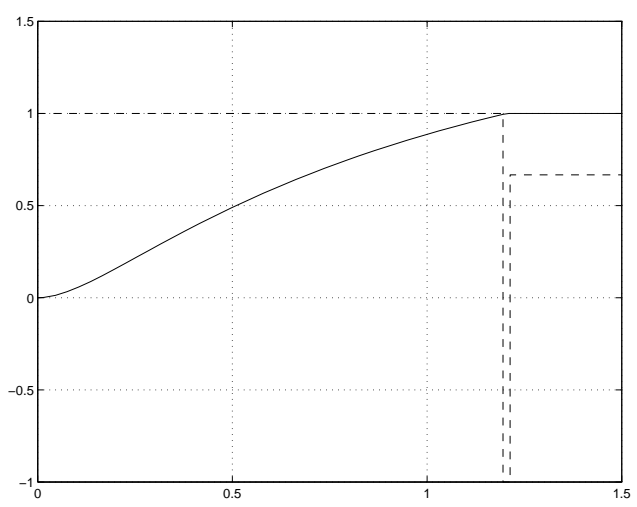

Figure 4: Minimum time step response. $G(0)=1.5, u_{0}=y_{0}=1$, time constants 1 and 0.1 . 


\section{Comparison with standard controllers}

It is also of interest to compare the performance with that obtained with standard industrial controllers of PI type, with anti-windup to take the input saturation into account. The responses of such a controller for the systems (30) and (31) are given in figures 5 and 6 respectively. In this case there is no definite
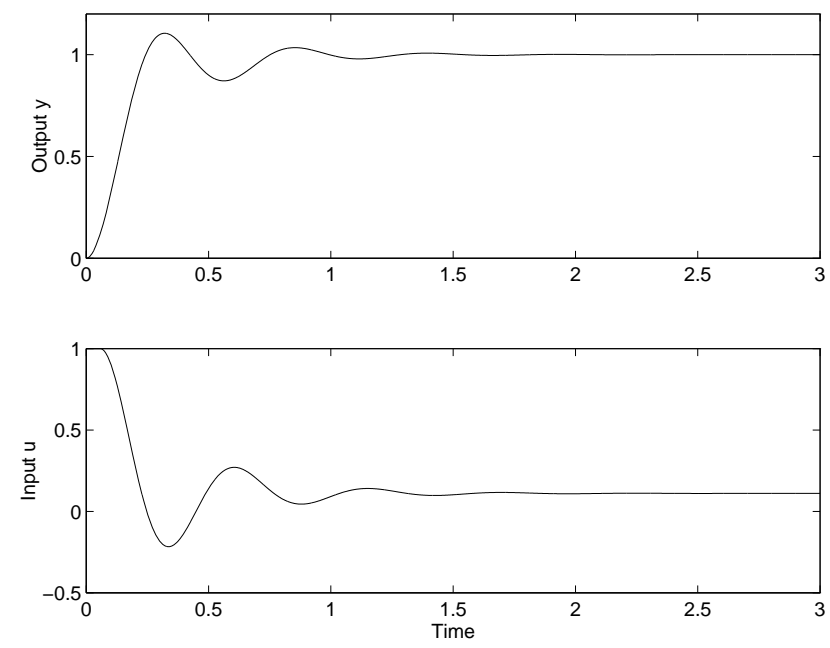

Figure 5: Step response of (30) under PI control with $u_{0}=y_{0}=1$.

final time $t_{f}$, since the new equilibrium is reached only asymptotically. We see that our computed lower bounds for $t_{f}(0.12$ and 1.1$)$ respectively provide a good estimate of the step response rise time.

\section{Conclusions}

With the formula

$$
t_{f} \geq-\tau \log \left(1-\frac{y_{0}}{G(0) u_{0}}\right)
$$

where $u_{0}$ is the control amplitude, $y_{0}$ is the step amplitude, and $\tau$ is the largest time constant, we have a practical lower bound of the duration of the step response. A similar formula gives a lower bound when $G$ has a pole at the origin. There are many extensions and refinements possible. The formula above does not apply if there is no real pole. Other extensions concern for instance the influence of unstable poles, asymmetries when the initial state is nonzero, and estimates of the influence of the shorter time constants.

\section{References}

[1] J. S. Freudenberg and D. P. Looze. Frequency Domain Properties of Scalar and Multivariable Feedback Systems. Springer-Verlag, New York, 1988. 

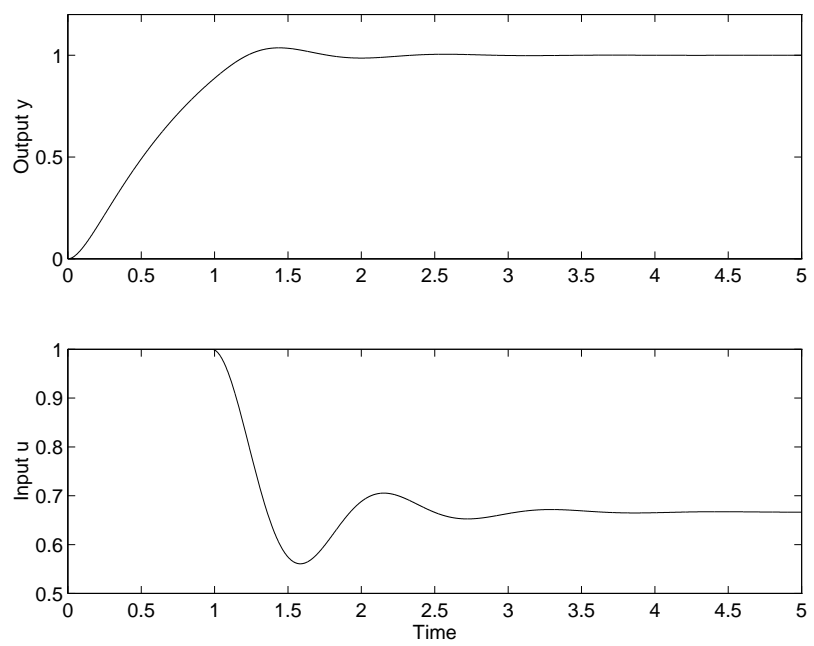

Figure 6: Step response of (31) under PI control with $u_{0}=y_{0}=1$.

[2] George Leitmann. The Calculus of Variations and Optimal Control. Plenum Press, 1981.

[3] J. M. Maciejowski. Multivariable Feedback Design. Addison-Wesley Publishing Company, Reading, MA, 1989.

[4] M. M. Seron, J. H. Braslavsky, and Graham C. Goodwin. Fundamental Limitations in Filtering and Control. Springer-Verlag, New York, 1997.

[5] S. Skogestad and I. Postlethwaite. Multivariable Feedback Control. John Wiley, New York, 1996. 